J. Appl. Numer. Optim. 2 (2020), No. 1, pp. 63-70

Available online at http://jano.biemdas.com

https://doi.org/10.23952/jano.2.2020.1.04

\title{
SUPERIORIZED REGULARIZATION OF INVERSE PROBLEMS
}

\author{
AVIV GIBALI ${ }^{1,2, *}$, MARKUS HALTMEIER $^{3}$ \\ ${ }^{1}$ Department of Mathematics, ORT Braude College, Karmiel 2161002, Israel \\ ${ }^{2}$ The Center for Mathematics and Scientific Computation, University of Haifa, Mt. Carmel, Haifa 3498838, Israel \\ ${ }^{3}$ Department of Mathematics, University of Innsbruck, Technikerstrasse 13, 6020 Innsbruck, Austria
}

\begin{abstract}
Inverse problems are characterized by their inherent non-uniqueness and sensitivity with respect to data perturbations. Their stable solution requires the application of regularization methods including variational and iterative regularization methods. Superiorization is a heuristic approach that can steer basic iterative algorithms to have small value of a certain regularization functional while keeping the algorithms simplicity and computational efforts, and is able to account for additional prior information. In this note, we combine the superiorization methodology with iterative regularization and show that the superiorized version of the scheme yields again a regularization method, however accounting for different prior information.
\end{abstract}

Keywords. Inverse problems; Iterative regularization; Generalized inverse; Superiorization.

\section{INTRODUCTION}

Throughout this paper, let $\mathbb{X}$ and $\mathbb{Y}$ be Hilbert spaces and let $\mathbf{A}: \mathbb{X} \rightarrow \mathbb{Y}$ be a bounded linear operator. We study the stable solution of the inverse problem of estimating the unknown $x \in \mathbb{X}$ from data

$$
y^{\delta}=\mathbf{A}(x)+\xi^{\delta},
$$

where $\xi^{\delta}$ models the noise (data error) that we assume to satisfy the noise estimate $\left\|\xi^{\delta}\right\| \leq \delta$ with noise level $\delta \geq 0$. In particular, we are interested in the ill-posed case, where solutions of (1.1) are non-unique (even for exact data), are unstable with respect to data perturbations, or suffer from both issues. Several practically relevant applications can be formulated in such form, including computed tomography, geophysical imaging, or nondestructive testing [11, 18, 19]. In order to account for the ill-posedness of (1.1) one has to apply regularization methods, which enforce uniqueness by using a suitable right inverse of $\mathbf{A}$, and stabilize the inversion by relaxing the exact solution concept.

Classical regularization methods use approximations of the Moore-Penrose inverse, which is the right inverse defined by selecting the solution of $\mathbf{A} x=y$ with minimal Hilbert space norm. Various variational and iterative regularization methods fit this into this framework. In

\footnotetext{
${ }^{*}$ Corresponding author.
}

E-mail addresses: avivg@braude.ac.il (A. Gibali), markus.haltmeier@uibk.ac.at (M. Haltmeier).

Received February 20, 2020; Accepted March 27, 2020.

(C)2020 Journal of Applied and Numerical Optimization 
particular, the Landweber method $[11,17]$ which uses the iterative update

$$
x_{k+1}^{\delta}=x_{k}^{\delta}-\lambda \mathbf{A}^{*}\left(\mathbf{A} x_{k}^{\delta}-y^{\delta}\right)
$$

( $\mathbf{A}^{*}$ denotes the adjoint of $\mathbf{A}$ ) with starting value $x_{0}^{\delta}=0$ is probably the most established iterative regularization method based on the Moore-Penrose inverse. The Landweber method can be seen as gradient based iteration applied to the least squares functional $\frac{1}{2}\left\|\mathbf{A} x-y^{\delta}\right\|^{2}$ combined with a suitable stopping rule in the case of noisy data. For exact data $y \in \mathbf{A}(\mathbb{X})$, the Landweber iteration converges to the Moore-Penrose inverse applied to exact data, which is equal to the minimal norm solution $\mathbf{A}^{+}(y)=\arg \min _{x}\{\|x\| \mid \mathbf{A} x=y\}$. For noisy data, the iterates $x_{k}^{\delta}$ define a regularization method in sense that together with a suitable stopping rule $k=\kappa(\delta)$ we have $\left\|x_{\kappa(\delta)}^{\delta}-\mathbf{A}^{+}(y)\right\| \rightarrow 0$ as $\delta \rightarrow 0$.

Minimal Hilbert space norm solutions, however, are often not the relevant ones in practical application. For example, signals of interest may be characterized by having small values of some regularizer $r: \mathbb{X} \rightarrow[0, \infty]$ such as the $\ell_{1}$-norm with respect to a certain frame, or the total variation (see, for example, [1, 4, 5, 12]).

Generalizing regularization methods based on the Moore-Penrose inverse consider approximations of $r$-minimizing solutions, that are elements in $\arg \min _{x}\{r(x) \mid \mathbf{A} x=y\}$. However, iterative algorithms implementing such approaches are typically more complex than gradient type methods such as (1.2).

In this paper, we therefore follow a different approach, where instead of iterative algorithms aiming for strictly minimizing $r(x)$ over the set of all solution of $\mathbf{A} x=y$, we modify (1.2) such that it reduces the value of the regularizer opposed to the basic iteration. More precisely, we define

$$
\left\{\begin{array}{l}
x_{k+1 / 2}^{\delta}=x_{k}^{\delta}+t_{k} \Phi_{k}\left(x_{k}^{\delta}\right), \\
x_{k+1}^{\delta}=x_{k+1 / 2}^{\delta}-\lambda \mathbf{A}^{*}\left(\mathbf{A} x_{k+1 / 2}^{\delta}-y^{\delta}\right)
\end{array}\right.
$$

where $\Phi_{k}(\cdot)$ are continuous perturbations with bounded range and $t_{k}$ is a summable sequence of non-negative numbers. The perturbations are chosen such that: $r\left(x_{k+1 / 2}^{\delta}\right) \leq r\left(x_{k}^{\delta}\right)$, meaning that the perturbed update has no larger value of $r$ than the unperturbed update. In that context one refers to (1.3) as the superiorized version of (1.2).

The concept of superiorization is first introduced (under a different name) in [2] and studied further by many authors is various fields and applications. A main ingredient in these works is the perturbation resilience in the case of exact data $[8,10]$. For the state of current research on superiorization one can check the website [7]. In particular, see [9, 13, 14, 15, 20] for some recent papers on superiorization in the context of least squares minimization including (1.2).

The aim of this paper is to analyze superiorization for ill-posed problems and to study its convergence behavior as $\delta \rightarrow 0$. As we shall show, the iteration (1.3) again defines a regularization method, which however does not approximate the Moore-Penrose inverse but a more general right inverse, that can be adapted to desired properties of solutions of $\mathbf{A} x=y$. To the best of our knowledge superiorization has not been previously analyzed as regularization method. We have selected the Landweber method as prime iterative regularization method but are convinced that many other superiorized iterative algorithms can be shown to yield regularization methods. 


\section{PRELIMINARIES}

In this section, we recall the concepts of regularization methods, perturbation resilience and the superiorization methodology in the Hilbert space context. Moreover, we derive some auxiliary results that we require for later purpose.

2.1. Regularization methods. Let $\mathbf{R}: \mathbf{A}(\mathbb{X}) \subseteq \mathbb{Y} \rightarrow \mathbb{X}$ be a right inverse of $\mathbf{A}$, which means any possibly non-linear mapping such that $\mathbf{A} \circ \mathbf{R}(y)=y$ for all $y \in \mathbf{A}(\mathbb{X})$.

For exact data, the selection of a particular right inverse defines a unique solution concept for the equation $\mathbf{A} x=y$. However, as the following Proposition 2.1 states, in the case that $\mathbf{A}$ has non-closed range, then any right inverse is unbounded. For example, any compact operator has non-closed range including integral operators arising in typical inverse problems. This is the reason for using regularizations methods as stable approximations of right inverses.

Proposition 2.1. If $\mathbf{A}(\mathbb{X})$ is non-closed, then $\mathbf{R}$ is discontinuous.

Proof. Every solution the equation $\mathbf{A} x=y$ has the form $\mathbf{A}^{+}(y)+z$ where $z \in \operatorname{ker}(\mathbf{A})$. In particular, we have $\mathbf{P}_{\operatorname{ker}(\mathbf{A}) \perp} \mathbf{R}=\mathbf{A}^{+}$. This shows that the continuity of $\mathbf{R}$ implies the continuity of $\mathbf{A}^{+}$. However, the continuity of $\mathbf{A}^{+}$implies the closeness of $\mathbf{A}(\mathbb{X})$ (see for example [11]) and concludes the proof.

Hence in the ill-posed setting where $\mathbf{A}$ has non-closed range, any right inverse is discontinuous. For the stable solution of such inverse problems one has to apply regularization methods that are defined as follows.

Definition 2.1 (Regularization method). A family $\left(\mathbf{R}_{k}\right)_{k \in \mathbb{N}}$ of continuous operators $\mathbf{R}_{k}: \mathbb{Y} \rightarrow \mathbb{X}$ is called regularization of $\mathbf{R}$ if for all $y \in \mathbf{A}(\mathbb{X})$ there exists a mapping $\kappa:(0, \infty) \rightarrow \mathbb{N}$ with $\kappa(\delta) \rightarrow \infty$ as $\delta \rightarrow 0$ and

$$
\lim _{\delta \rightarrow 0} \sup \left\{\left\|\mathbf{R}(y)-\mathbf{R}_{\kappa(\delta)}\left(y^{\delta}\right)\right\| \mid y^{\delta} \in \mathbb{Y} \wedge\left\|y^{\delta}-\mathbf{A} x\right\| \leq \delta\right\}=0 .
$$

In this case we call $\kappa$ (a-priori) parameter choice rule and the pair $\left(\left(\mathbf{R}_{k}\right)_{k \in \mathbb{N}}, \kappa\right)$ a regularization method for the solution of $\mathbf{A} x=y$.

Classical regularization methods are adapted to the Moore-Penrose inverse $\mathbf{A}^{+}$where $\mathbf{A}^{+}(y)$ for $y \in \mathbf{A}(\mathbb{X})$ is defined as the unique solution of $\mathbf{A} x=y$ with minimal norm. This includes classical Tikhonov regularization and classical iterative regularization methods. In particular, the Landweber iteration (1.2) together with a suitable stopping rule is known to be a regularization method. In this work we generalize these results by integrating the superiorization methodology to the Landweber iteration and thereby adapting to more flexible right inverses. The following lemma gives a useful guideline for creating regularization methods.

Proposition 2.2 (Point-wise approximations are regularizations). Let $\left(\mathbf{R}_{k}\right)_{k \in \mathbb{N}}$ be a family of continuous operators $\mathbf{R}_{k}: \mathbb{Y} \rightarrow \mathbb{X}$ that converge point-wise to $\mathbf{R}$ on $\mathbf{A}(\mathbb{X})$. Then $\left(\mathbf{R}_{k}\right)_{k \in \mathbb{N}}$ is a regularization of $\mathbf{R}$.

Proof. We follow the proof given in [11] for the special case $\mathbf{R}=\mathbf{A}^{+}$. Let $y \in \mathbf{A}(\mathbb{X})$. For any $\varepsilon>0$ choose $k(\varepsilon)$ such $\left\|\mathbf{R}_{k(\varepsilon)}(y)-\mathbf{R}(y)\right\| \leq \varepsilon / 2$. Moreover, choose $\tau(\varepsilon)$ such that for all $z \in \mathbb{Y}$ with $\|y-z\| \leq \tau(\varepsilon)$ we have $\left\|\mathbf{R}_{k(\varepsilon)}(y)-\mathbf{R}_{k(\varepsilon)}(z)\right\| \leq \varepsilon / 2$. Without loss of generality, we can 
assume that $\tau(\varepsilon)$ is strictly increasing and continuous with $\tau(0+)=0$. We define $\kappa:=k \circ \tau^{-1}$. Then, for every $\delta>0$ and $\left\|y-y^{\delta}\right\| \leq \delta$,

$$
\begin{aligned}
\left\|\mathbf{R}_{\kappa(\delta)}\left(y^{\delta}\right)-\mathbf{R}(y)\right\| & \leq\left\|\mathbf{R}_{\kappa(\delta)}(y)-\mathbf{R}(y)\right\|+\left\|\mathbf{R}_{\kappa(\delta)}(y)-\mathbf{R}_{\kappa(\delta)}\left(y^{\delta}\right)\right\| \\
& =\left\|\mathbf{R}_{k \circ \tau^{-1}(\delta)}(y)-\mathbf{R}(y)\right\|+\left\|\mathbf{R}_{k \circ \tau^{-1}(\boldsymbol{\delta})}(y)-\mathbf{R}_{k \circ \tau^{-1}(\delta)}\left(y^{\delta}\right)\right\| \\
& \leq \tau^{-1}(\boldsymbol{\delta}) / 2+\tau^{-1}(\boldsymbol{\delta}) / 2 \\
& =\tau^{-1}(\boldsymbol{\delta}) .
\end{aligned}
$$

Since $\tau^{-1}(\delta) \rightarrow 0$ as $\delta \rightarrow 0$, this completes the proof.

Proposition 2.2 will be used to show that superiorized Landweber method is a regularization method.

2.2. Superiorization. Let $\mathbb{S} \subseteq \mathbb{X}$ be a given set, $\mathbf{T}: \mathbb{X} \rightarrow \mathbb{X}$ be an algorithmic operator for the problem of finding elements in $\mathbb{S}$, and suppose the basic iteration

$$
x_{k+1}=\mathbf{T}\left(x_{k}\right)
$$

converges for all $x_{0} \in \mathbb{X}$ to some element in $\mathbb{S}$. The superiorization methodology modifies (2.2) such that the resulting iteration still converges, however with a limit having lower or equal value of some functional $r: \mathbb{X} \rightarrow[0, \infty]$. Superiorization comes with several benefits. First, limits having small value of $r$ are often closer to desired elements than the original limits of (2.2). Second, one can make use of existing algorithms in the form of (2.2). Third, opposed to optimization problems that strictly minimize the functional $r$, superiorized algorithms are often simpler and more efficient to implement. In this context we also mention that exactly minimizing the regularizer $r$ is anyway not strictly required in many inverse problems because the selection of the regularizer itself is often somehow heuristic.

The superiorized version of (2.2) is defined as follows.

Definition 2.2. Let $\mathbb{S} \subseteq \mathbb{X}$, let (2.2) for all $x_{0} \in \mathbb{X}$ converge to an element in $\mathbb{S}$, and let $r: \mathbb{X} \rightarrow$ $[0, \infty]$ be convex and subdifferentiable. One calls $x_{k+1}=\mathbf{T}\left(x_{k}+t_{k} d_{k}\right)$, where $t_{k} \geq 0$ with $\sum_{k \in \mathbb{N}} t_{k}<\infty$, and

$$
d_{k}:= \begin{cases}-D_{k} /\left\|D_{k}\right\|, & \text { if } D_{k} \neq 0, \\ 0, & \text { otherwise }\end{cases}
$$

with $D_{k} \in \partial r\left(x_{k}\right)$, the superiorized version of (2.2).

In Definition 2.2 we used the superiorization strategy based on the subgradient. This approach can be generalized by using further superiorization strategies, such as derivatives free techniques, see also [9] in the context of least squares minimization. Note that for the presented convergence analysis we assume that perturbations have the form $d_{k}=\Phi_{k}\left(x_{k}\right)$ with continuous $\Phi_{k}(\cdot)$. This can be achieved by smoothing the normalization procedure in (2.3) around zero.

General questions concerning the superiorization methodology are the following:

(a) Does the superiorized iteration converge?

(b) Is the limit $\hat{x}$ contained $\mathbb{S}$ ?

(c) Is $r(\hat{x})$ not larger than $r$ evaluated at the limit of the basic sequence? 
The underlying concept addressing the issues (a), (b) is perturbation resilience that is defined next. That limit points satisfy (c) is referred to as the guarantee problem of the superiorization methodology and could not be proven until today.

Definition 2.3. Let $\mathbb{S} \subseteq \mathbb{X}$ and suppose that for all initial values $x_{0} \in \mathbb{X}$ the sequence (2.2) converges to some element in $\mathbb{S}$. Iteration (2.2) is called bounded perturbation resilient (with respect to $\mathbb{S}$ ), if for all $x_{0} \in \mathbb{X}$, all $\left(t_{k}\right)_{k \in \mathbb{N}} \in[0, \infty)^{\mathbb{N}}$ with $\sum_{k \in \mathbb{N}} t_{k}<\infty$ and all bounded sequences $\left(d_{k}\right)_{k \in \mathbb{N}} \in \mathbb{X}^{\mathbb{N}}$, the perturbed iteration

$$
x_{k+1}=\mathbf{T}\left(x_{k}+t_{k} d_{k}\right)
$$

converges to some element in $\mathbb{S}$.

The following result which is a direct consequence of [3, Theorem 5] shows that the first two questions in the above list can be positively answered for a wide class of basic iterations.

Proposition 2.3. Let $\mathbf{T}$ be non-expansive and all basic iterates (2.2) converge strongly to some element $\operatorname{Fix}(\mathbf{T}) \neq \emptyset$. Then (2.2) is bounded perturbation resilient.

Proof. Under the given assumptions, [3, Theorem 5] states that any sequence $\left(z_{k}\right)_{k \in \mathbb{N}}$ satisfying $\sum_{k \in \mathbb{N}}\left\|z_{k+1}-\mathbf{T}\left(z_{k}\right)\right\|<\infty$ converges to a fixed point of $\mathbf{T}$. Because of the non-expansiveness of $\mathbf{T}$, the iteration (2.4) satisfies $\sum_{k \in \mathbb{N}}\left\|x_{k+1}-\mathbf{T}\left(x_{k}\right)\right\| \leq \sup _{k}\left\|d_{k}\right\| \sum_{k \in \mathbb{N}} t_{k}$ which allows applying above mentioned result.

We will apply Proposition 2.3 to show the perturbation resilience of the Landweber iteration for exact data. In that context, we will also discuss regularizing properties of the superiorized Landweber iteration for noisy data, which, to the best of our knowledge, has not been investigated so far.

\section{CONVERGENCE ANALYSis}

Recall that $\mathbf{A}: \mathbb{X} \rightarrow \mathbb{Y}$ is a bounded linear operator and choose $\lambda \in\left(0,1 /\|\mathbf{A}\|^{2}\right)$. Moreover, let $\Phi_{k}: \mathbb{X} \rightarrow \mathbb{X}$ be a family of continuous mappings with bounded range and let $\left(t_{k}\right)_{k \in \mathbb{N}}$ be a summable sequence of nonnegative numbers.

We investigate the perturbed Landweber iteration for possibly noisy data that is defined as follows:

$$
\left\{\begin{array}{l}
x_{k+1 / 2}^{\delta}=x_{k}^{\delta}+t_{k} \Phi_{k}\left(x_{k}^{\delta}\right), \\
x_{k+1}^{\delta}=x_{k+1 / 2}^{\delta}-\lambda \mathbf{A}^{*}\left(\mathbf{A} x_{k+1 / 2}^{\delta}-y^{\delta}\right),
\end{array}\right.
$$

with initial data $x_{0}^{\delta}=0$. For properties of the Landweber operator the readers are referred to the work of [6]. The index $\delta \geq 0$ stands for the noise level and the given data $y^{\delta}$ satisfy the estimate $\left\|y-y^{\delta}\right\| \leq \delta$ with $y \in \mathbf{A}(\mathbb{X})$. To indicate the dependence of the iterates on the given data we write $\mathbf{B}_{k}\left(y^{\delta}\right):=x_{k}^{\delta}$.

3.1. Exact data. Our aim is to show that (3.1) defines a regularization method. We first start with the convergence in the exact data case.

Theorem 3.1 (Convergence for exact data). The Landweber iteration (1.2) is perturbation resilient. That is, for all $x_{0} \in \mathbb{X}$, the perturbed Landweber iteration with exact data (3.1) converges to a solution of the equation $\mathbf{A}(x)=y$. In particular, the limits of the iteration (3.1) define a right inverse $\mathbf{B}: \mathbf{A}(\mathbb{X}) \rightarrow \mathbb{Y}: y \mapsto \lim _{k \rightarrow \infty} \mathbf{B}_{k}(y)$ of $\mathbf{A}$. 
Proof. The basic Landweber iteration is for exact data known to strongly converge to a solution of $\mathbf{A}(x)=y$ (see for example [11, Theorem 6.1]). Moreover, note that (3.1) is a perturbed fixed point iteration with the operator $\mathbf{T}(x)=\left(\operatorname{Id}-\lambda \mathbf{A}^{*} \mathbf{A}\right)(x)+\mathbf{A}^{*} y$. We have

$$
\left\|\mathbf{T}(x)-\mathbf{T}\left(x_{0}\right)\right\| \leq\left\|\mathrm{Id}-\lambda \mathbf{A}^{*} \mathbf{A}\right\|\left\|x-x_{0}\right\| \leq\left\|x-x_{0}\right\| .
$$

Hence $\mathbf{T}$ is non-expansive and because $y \in \mathbf{A}(\mathbb{X})$, we have $\operatorname{Fix}(\mathbf{T})=\{x \mid \mathbf{A} x=y\}$. Therefore the claim follows from Proposition 2.3.

3.2. Noisy data. Proposition 2.1 implies that in the ill-posed case where $\mathbf{A}(\mathbb{X})$ is non-closed, the right inverse $\mathbf{B}$ defined by the perturbed Landweber exact data iteration (3.1) is discontinuous. Therefore, it has to be regularized. Following the iterative regularization strategy, the regularization we use in this paper comes from early stopping the noisy data iteration. Recall that we write $\mathbf{B}_{k}\left(y^{\delta}\right)=x_{k}^{\delta}$ for the iterates defined in (3.1), defining mappings $\mathbf{B}_{k}: \mathbb{Y} \rightarrow \mathbb{X}$.

Theorem 3.2 (Convergence for noisy data). For all $y \in \mathbf{A}(\mathbb{X})$ there exists a parameter choice rule $\kappa:(0, \infty) \rightarrow \mathbb{N}$ such that the pair $\left(\left(\mathbf{B}_{k}\right)_{k \in \mathbb{N}}, \kappa\right)$ is a regularization method for the solution of $\mathbf{A} x=y$ adapted to $\mathbf{B}$. In particular,

$$
\lim _{\delta \rightarrow 0}\left\|\mathbf{B}(y)-x_{\kappa(\delta)}^{\delta}\right\|=0
$$

for all families of noisy data $\left(y^{\delta}\right)_{\delta>0}$ with $\left\|y-y^{\delta}\right\| \leq \delta$. Moreover, the truncated iterates form a regularization $\left(\mathbf{B}_{k}\right)_{k \in \mathbb{N}}$ of $\mathbf{B}$.

Proof. For exact data, according to Theorem 3.1, we have $\lim _{k \rightarrow \infty} \mathbf{B}_{k}(y)=\mathbf{B}(y)$ pointwise for $y \in \mathbf{A}(\mathbb{X})$. Moreover, in an inductive manner one verfies that the mappings $\mathbf{B}_{k}: \mathbb{Y} \rightarrow \mathbb{X}$ are continuous for all $k \in \mathbb{N}$. Proposition 2.2 therefore implies the existence of $\kappa:(0, \infty) \rightarrow \mathbb{N}$ such that the pair $\left(\left(\mathbf{B}_{k}\right)_{k \in \mathbb{N}}, \kappa\right)$ is a regularization method for the solution of $\mathbf{A} x=y$ adapted to $\mathbf{B}$. In particular, (3.2) holds and $\left(\mathbf{B}_{k}\right)_{k \in \mathbb{N}}$ is a regularization of $\mathbf{B}$.

Remark 3.1. Theorem 3.2 shows that there exists a parameter choice rule which yields a regularization method, but this is not given explicitly. In order to define this parameter choice rule, further investigation and additional prior information is needed; for example one can explore the following strategies.

- Estimate the Lipschitz constant of $B_{k}$ to find a-priori rules $k=\kappa(\delta)$.

- Show that the discrepancy principle gives a-posteriori rule $k=\kappa\left(\delta, y^{\delta}\right)$.

For the second strategy, relations between the discrepancy principle and the concept of strong perturbation resilience might be useful [16].

\section{CONCLUSION}

In this paper we showed that the superiorization concept applied to the Landweber method gives a regularization method for the solution of inverse problems. Basically, our main result states that truncating the superiorized (or perturbed) Landweber iteration depending on the noise level, is stable and convergent in the limit $\delta \rightarrow 0$. To the best of our knowledge, such regularization properties have not been investigated previously for the superiorization methodology.

However, many relevant questions following this work regarding superiorization and regularization remain open and call for further investigations.

1: Explicit parameter choice rules must be derived. 
2: For the Landweber (and many related regularization techniques), the discrepancy principle which chooses the first index with $\left\|\mathbf{A} x_{k}^{\delta}-y^{\delta}\right\| \leq c \delta$ yields an admissible parameter choice. It is unclear if a similar parameter choice rule for the superiorized Landweber iteration exists.

3: The Landweber method is often quite slow. Accelerated iterative regularization might be investigated in combination with the superiorization technique.

4: Convergence rates (quantitative estimates between the exact solution $\mathbf{B} y$ for exact data and regularized solutions $\mathbf{B}_{k} y^{\delta}$ for noisy data) are well established for the Landweber iteration. Deriving such rates for the superiorized version seems a difficult issue. In that context characterizing $\mathbf{B} y$ might be useful.

5: Superiorization in the context of nonlinear inverse problems is another aspect that we have not touched.

Investigating such issues are interesting lines of future research.

\section{Acknowledgements}

The work of M. Haltmeier was supported by the Austrian Science Fund (FWF), project P 30747N32.

\section{REFERENCES}

[1] R. Acar, C. R. Vogel, Analysis of bounded variation penalty methods for ill-posed problems, Inverse Probl. 10 (1984), 1217-1229.

[2] D. Butnariu, R. Davidi, G. T. Herman, I. G. Kazantsev, Stable convergence behavior under summable perturbations of a class of projection methods for convex feasibility and optimization problems, IEEE J. Sel. Topics Signal Process. 1 (2007), 540-547.

[3] D. Butnariu, S. Reich, A. J. Zaslavski, Convergence to fixed points of inexact orbits of Bregman- monotone and of nonexpansive operators in Banach spaces, In: Proceedings of the Seventh International Conference on Fixed Point Theory and its Applications, pp. 11-32, Mexico, 2005.

[4] D. Calvetti, F Pitolli, E. Somersalo, B Vantaggi. Bayes meets Krylov: Statistically inspired preconditioners for cgls, SIAM Rev. 60 (2018), 429-461.

[5] E. J. Candès, D. Donoho, Recovering edges in ill-posed inverse problems: Optimality of curvelet frames, Ann. Statist. 30 (2002), 784-842.

[6] A. Cegielski, Landweber-type operator and its properties, Contemp. Math. 658 (2014), 139-148.

[7] Y. Censor, Superiorization and perturbation resilience of algorithms: A bibliography compiled and continuously updated. http://math.haifa.ac.il/yair/bib-superiorization-censor.html.

[8] Y. Censor, E. Garduño, E. S. Helou, G. T. Herman, Derivative-free superiorization: Principle and algorithm, arXiv:1908.10100, 2019.

[9] Y. Censor, S. Petra, C. Schnörr. Superiorization vs. accelerated convex optimization: The superiorized/regularized least-squares case, arXiv:1911.05498, 2019.

[10] R. Davidi, G. T. Herman, Y. Censor, Perturbation-resilient block-iterative projection methods with application to image reconstruction from projections, Int. Trans. Oper. Res. 16 (2009), 505-524.

[11] H. W. Engl, M. Hanke, A. Neubauer. Regularization of inverse problems, volume 375. Kluwer Academic Publishers Group, Dordrecht, 1996.

[12] M. Grasmair, M. Haltmeier, O. Scherzer. Necessary and sufficient conditions for linear convergence of $l^{1}$ regularization, Commun. Pure Appl. Math. 64 (2011), 161-182.

[13] Y. Guo, W. Cui, Strong convergence and bounded perturbation resilience of a modified proximal gradient algorithm, J. Inequal. Appl. 2018 (2012), 103.

[14] Y. Guo, X. Zhao, Bounded perturbation resilience and superiorization of proximal scaled gradient algorithm with multi-parameters, Mathematics, 7 (2019), 535. 
[15] E. S. Helou, G. T. Herman, C. Lin, M. V. W. Zibetti, Superiorization of preconditioned conjugate gradient algorithms for tomographic image reconstruction, arXiv:1807.10151, 2018.

[16] G. T. Herman, E. Garduño, R. Davidi, Y. Censor. Superiorization: An optimization heuristic for medical physics, Med. Phys. 39 (2012), 5532-5546.

[17] L. Landweber, An iteration formula for Fredholm integral equations of the first kind, Amer. J. Math. 73 (1951), 615-624.

[18] F. Natterer, F. Wübbeling, Mathematical Methods in Image Reconstruction, volume 5 of Monographs on Mathematical Modeling and Computation. SIAM, Philadelphia, PA, 2001.

[19] O. Scherzer, M. Grasmair, H. Grossauer, M. Haltmeier, F. Lenzen, Variational methods in imaging, volume 167 of Applied Mathematical Sciences, Springer, New York, 2009.

[20] M. V. W. Zibetti, C. Lin, G. T. Herman, Total variation superiorized conjugate gradient method for image reconstruction, Inverse Probl. 34 (2018), 034001. 